\title{
Anti-urolithiatic Activity of Sambong (Blumea balsamifera) Extract in Ethylene Glycol-induced Urolithiatic Wistar Rats (Rattus norvegicus)
}

\author{
Althea Samantha C. Agdamag, ${ }^{1}$ Larielyn Hope C. Aggabao, ${ }^{1}$ Mary Sheena C. Agudo, ${ }^{1}$ Francis Louis M. Alcachupas, ${ }^{1}$ \\ Jeremiah Carlo V. Alejo, ${ }^{1}$ Shari A. Altamera, ${ }^{1}$ Jose Nicolo D. Antonio, ${ }^{1}$ Jeric L. Arbizo, ${ }^{1}$ Jose Joaquin H. Arroyo, ${ }^{1}$ \\ Daniel Raphael D.G. Bañez, ${ }^{1}$ Vincent Bryan B. Balaong, ${ }^{1}$ Nicole Audri R. Belo, ${ }^{1}$ Noel L. Bernardo, ${ }^{1}$ \\ John Jefferson V. Besa, ${ }^{1}$ John Harvey M. Beza ${ }^{1}$ and Tammy L. Dela Rosa ${ }^{2}$ \\ ${ }^{1}$ College of Medicine, University of the Philippines Manila \\ ${ }^{2}$ Department of Biochemistry, College of Medicine, University of the Philippines Manila
}

\begin{abstract}
Objective. The study aimed to determine if Blumea balsamifera inhibits calcium oxalate stone formation in the kidneys through determination of the number of calcium oxalate stones in the renal cortex and the percent mass of calcium oxalate.

Methods. Post-test only control group design was used using five treatment groups with placebo as the negative control, potassium citrate as the positive control, and 50\%, 100\%, and $200 \%$ sambong treatment. Urolithiasis was induced through ethylene glycol and ammonium chloride. Each treatment group was administered its corresponding treatment solution once daily for twenty-one days. Histopathologic examination and kidney homogenate analysis were done to determine the degree of deposition of calcium oxalate stones in renal tissues and the oxalate content, respectively. Statistical analyses were performed using one-way ANOVA and post hoc Gabriel's Pairwise Comparisons Test.
\end{abstract}

Results. The $100 \%$ sambong treatment group showed the least mean number of stones while the positive control and $50 \%$ sambong treatment group exhibited the highest anti-urolithiatic activity in terms of oxalate content of the kidney homogenate.

Conclusion. It can be concluded from the study that Blumea balsamifera inhibits calcium oxalate stone formation in the kidneys with the $100 \%$ and $50 \%$ sambong treatment most effective in decreasing number of stones and oxalate content of the kidney homogenate, respectively.

Key Words: Sambong, Blumea balsamifera, urolithiasis, calcium oxalate stone, kidney

\section{INTRODUCTION}

Corresponding author: John Jefferson V. Besa College of Medicine

University of the Philippines Manila

547 Pedro Gil St. Ermita, Manila 1000, Philippines

Email: jvbesa@up.edu.ph
A kidney stone or renal calculus is a solid mass composed of crystals found in the kidney or ureter. ${ }^{1}$ In the Philippines, kidney disease is among the top prevalent noncommunicable diseases along with malignant neoplasms, chronic obstructive pulmonary disease, and diabetes mellitus. ${ }^{2}$

Presently, there are several treatments available in the form of medication or surgery. However, despite their availability, a few cost-effective and readily available remedies (e.g. herbal medicines) are said to offer a cure for kidney stones. An herbal medicine which refers to the use of a plant's seeds, roots, bark, leaves, flowers or berries for 
medicinal purposes is becoming increasingly popular due to its efficacy and cost-efficiency. ${ }^{3}$ Currently, Blumea balsamifera (L.) DC $>$ (family Asteraceae/ Compositae) or sambong is the most well-known herb used to dissolve kidney stones. Studies showed the successful dissolution of kidney stones by addition of sambong extract in vitro. ${ }^{4}$

Despite these positive claims, no in vivo study in rats has been conducted as of date to fully verify the antiurolithiasis property of sambong thus, the significance of this research. Proving the effectiveness of this medicinal plant as an anti-urolithiasis agent in vivo could provide a less costly preventive and treatment option for kidney stones among Filipinos due to its easy availability. Moreover, having said that no preliminary in vivo study in rats has been performed, this study could be used as a springboard for more in-depth studies of higher institutions. This study determined the anti-urolithiasis activity of different concentrations of sambong (Blumea balsamifera) extract on ethylene glycol-induced urolithiatic Sprague-Dawley rats (Rattus norvegicus).

\section{MATERIALS AND METHODS}

Post-test only control group design was employed in the experiment. A total of thirty-two (32) male hooded Wistar rats (Rattus norvegicus) were taken care of based on the guidelines of the University of the Philippines Manila - Research Ethics Board and approval of the Institutional Animal Care \& Use Committee of the University of the Philippines Manila - National Institutes of Health. After seven days of acclimatization, induction of urolithiasis was initiated in all rats using ethylene glycol $(0.75 \% \mathrm{v} / \mathrm{v})$ with ammonium chloride $(2 \% \mathrm{w} / \mathrm{v})$. It was initially done by allowing ad libitum access to drinking water containing the said mixture. ${ }^{5}$ However, review of the rats' water intake showed a reduction from acclimatization values thus the solution was given through oral gavage instead. The induction of urolithiasis lasted until the termination of the experiment.

Fresh sambong leaves were procured and were oven dried at low heat $\left(82.2^{\circ} \mathrm{C}\right)$ until the leaves were brittle. Preparation of the three sambong decoctions was based on the recommendation of the Department of Health. The first decoction (Decoction A) contains half the concentration of the recommended amount of leaves, i.e. two (2) tablespoons of dried leaves in $250 \mathrm{~mL}$ distilled water. The second (Decoction B) was the same as the DOH's recommended preparation i.e. four (4) tablespoons of dried sambong leaves. The third decoction (Decoction C) contains twice the recommended concentration, i.e. eight (8) tablespoons of dried sambong leaves.

After ten (10) days of initial induction of urolithiasis, it was assumed that the almost equal amount of the mixture given through oral gavage induced a comparable number of stones; it is impossible to quantitatively determine baseline stone formation as this would require killing the rats. The surviving twenty-nine (29) rats were randomized into five (5) treatment groups as shown in Table 1 . Potassium citrate and three sambong decoctions of varying concentrations were administered through oral feeding twice daily to the positive control group and to the three treatment groups (T1, T2, T3), respectively. Treatments were given for twenty-one (21) days.

Table 1. Treatment Groups

\begin{tabular}{ll}
\hline Treatment group & Treatment \\
negative control $[\mathrm{T}(-)]$ & none \\
positive control $[\mathrm{T}(+)]$ & $0.06 \mathrm{mEq}$ of potassium citrate \\
$50 \%$ sambong treatment group $\left(\mathrm{T}_{1}\right)$ & $0.50 \mathrm{~mL}$ of Decoction A \\
$100 \%$ sambong treatment group $\left(\mathrm{T}_{2}\right)$ & $0.50 \mathrm{~mL}$ of Decoction $\mathrm{B}$ \\
$200 \%$ sambong treatment group $\left(\mathrm{T}_{3}\right)$ & $0.50 \mathrm{~mL}$ of Decoction C \\
\hline
\end{tabular}

All animals from each treatment group were sacrificed at the end of the experiment. The right kidneys were used for histopathologic examination and the left kidney was used for homogenate analysis of oxalate content.

The right kidneys were collected and fixed in 10\% (v/v) formalin solution ( $\mathrm{pH}$ 7.4-7.6) overnight. Tissue samples were delivered to the Department of Pathology, University of the Philippines Manila College of Medicine to be dehydrated in ethanol, embedded in paraffin, and sectioned into $6 \mu \mathrm{m}$ serial sections. ${ }^{6}$ The tissues were stained using Pizzolato's Method for Calcium Oxalate for histological examination of calcium oxalate crystal formation. Calcium oxalate crystal formation was assessed under oil immersion objective (1000X). One field per rat kidney section was photographed and crystals were quantified using ImageJ software, an open source Java image processing program whose variability, accuracy, and traceability in automatically counting particles have been well described. ${ }^{7}$ Kidney field samples for all specimens were obtained from renal cortical areas with the greatest amount of crystal deposition. The assessors were blinded to which treatment groups the tissue samples belong by assigning coded designations to each sample unknown to the assessors.

The left kidneys were dried at $80^{\circ} \mathrm{C}$ in a hot air oven for 24 hours and weighed individually. Each kidney was homogenized individually and pooled within each treatment group. Then, each pooled sample was then dissolved in 10 $\mathrm{mL}$ of $1 \mathrm{~N} \mathrm{HCl}$ and heated in a water bath at $70^{\circ} \mathrm{C}$ for 1 hour. The solution was centrifuged at $2000 \mathrm{x}$ g for 10 minutes to separate the supernatant. Oxalate content in the kidney homogenate was determined through titrimetric analysis. Oxalate was precipitated first by adding $0.5 \mathrm{~mL}$ of $1 \mathrm{M} \mathrm{CaCl}_{2}$ solution and left overnight at $40^{\circ} \mathrm{C}$. Finally, it was titrated with $0.2 \mathrm{~N} \mathrm{KMnO}_{4}$ while keeping the solution at $90^{\circ} \mathrm{C}$ to determine the concentration of oxalate present in the homogenate. From the stoichiometric ratio of 2 $\mathrm{MnO}_{4}^{-}$is to 5 oxalates (2:5), the concentration of oxalate 
in the sample was determined. The concentration of calcium oxalate was reported as moles of calcium oxalate per gram of sample. This value was then multiplied by the molar mass of calcium oxalate. The obtained mass of calcium oxalate was reported in percent mass calcium oxalate in reference to the total weight of the dried kidney sample titrated. ${ }^{8,9}$

Laboratory activities were terminated once the necessary data from the kidney specimens were collected. The rats were discarded properly and were surrendered to the laboratory aide for proper disposal.

Experimental data were analyzed by one-way ANOVA using PASW Statistics software (SPSS, IBM Statistics) and post hoc Gabriel's Pairwise Comparison Test to compare significant differences in the amount of cortical oxalate crystals present after treatment of different concentrations of the sambong extract and the control groups and the amount of oxalate in the kidneys upon titrimetric analysis. Results were expressed as means and standard errors. P-values less than 0.05 were considered statistically significant. Effect size $\left(\eta^{2}\right)$ was also obtained to measure how much of the variability in treatment effects is influenced by the concentration of the plant extract and the plant itself.

\section{RESULTS}

Initially, thirty-two (32) male Hooded Wistar rats were utilized in the study. At the end of the experimentation, twenty-two (22) rats were able to survive the treatment period. Three (3) and seven (7) rats died during the period of induction of urinary crystals and treatment period, respectively. Of the seven (7) rats, three (3) came from the $200 \%$ sambong treatment group and one from each of the remaining groups. After autopsy of a randomly chosen rat, dehydration was hypothesized as the cause of death.

After histologic examination of the kidneys, results showed the formation of kidney calcium oxalate stones as shown in Figure 1. Using oil immersion objective, renal cortical tissue areas having the greatest amount of oxalate crystals were sampled for each specimen.

The number of oxalate crystals found in each area sampled from each kidney specimen was counted using ImageJ software. Table 2 shows the mean number of cortical crystals for each treatment group $F(4,17)=12.041, p<0.05$, $\eta=0.739$. Among the treatment groups, $100 \%$ sambong treatment had the lowest mean number of oxalate crystals

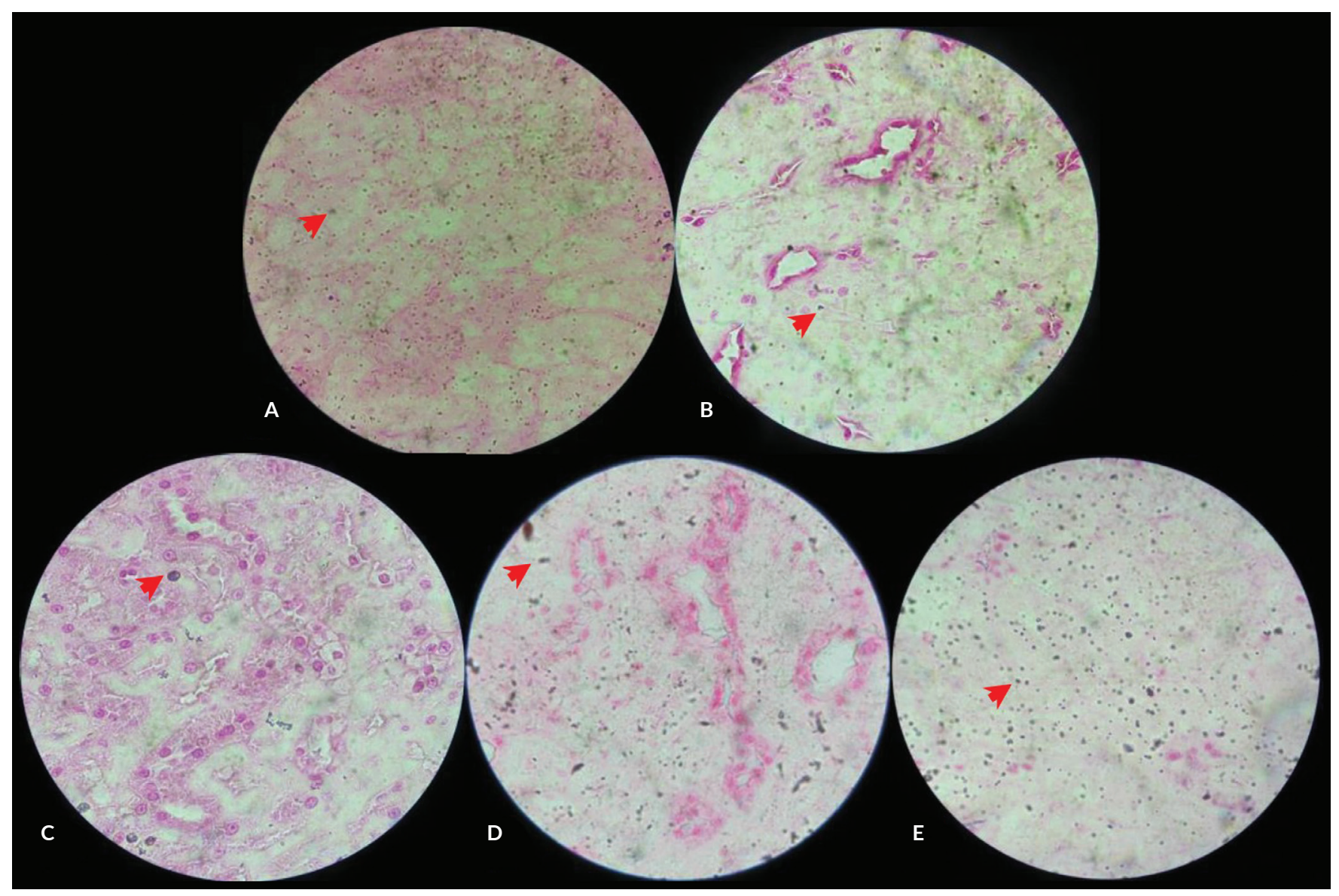

Figure 1. Histological sections of representative kidneys under the different treatment groups after Pizzolato staining (x1000). Black dots (red arrowheads) represent calcium oxalate stones. (A) negative control, (B) positive control, (C) $50 \%$ sambong, (D) $100 \%$ sambong, (E) $200 \%$ sambong. 
Table 2. Sample Size, Mean Number, And Range Of Cortical Crystals Per Treatment Group

\begin{tabular}{|c|c|c|}
\hline & $\mathbf{N}$ & Mean (Range) \\
\hline Negative control & 4 & $699.25(508-944)$ \\
\hline $50 \%$ sambonga & 5 & $315.60(125-417)$ \\
\hline $100 \%$ sambonga & 5 & $296.60(151-426)$ \\
\hline $200 \%$ sambong & 3 & $739.00(596-933)$ \\
\hline Positive control (potassium citrate) ${ }^{a}$ & 5 & $319.80(227-400)$ \\
\hline Total & 22 & $439.73(125-944)$ \\
\hline
\end{tabular}

a Statistically significantly different compared to negative control

Table 3. Titration Data Showing the Volumes of Titrant Used for each Treatment and the Calculated Percent Mass Calcium Oxalate

\begin{tabular}{lccc} 
& $\begin{array}{c}\text { Titrant } \\
\text { volume } \\
(\boldsymbol{\mu L})\end{array}$ & $\begin{array}{c}\text { Mass of } \\
\text { sample } \\
(\mathbf{g})\end{array}$ & $\begin{array}{c}\text { Percent mass calcium } \\
\text { oxalate in dried sample } \\
(\%)\end{array}$ \\
\hline Negative Control & 250 & 0.025 & 12.81 \\
Positive Control & 250 & 0.042 & 7.62 \\
50\% Treatment & 550 & 0.119 & 5.92 \\
100\% Treatment & 300 & 0.051 & 7.54 \\
200\% Treatment & 500 & 0.065 & 9.85 \\
\hline
\end{tabular}

( $M=296.60)$ while the $200 \%$ sambong treatment had the greatest mean number of oxalate crystals $(M=739)$ which is even greater than the mean of the negative control group $(\mathrm{M}=699.25)$. The 50\% sambong treatment had the specimen with the lowest number of crystals (125) while the negative control had the highest number of crystals (944).

Post hoc Gabriel's test revealed that the number of crystals in the negative control differed significantly from the $50 \%(\mathrm{p}=0.003)$ and $100 \%(\mathrm{p}=0.002)$ sambong treatments and the positive control $(\mathrm{p}=0.004)$ but did not significantly differ from the $200 \%$ sambong treatment. On the other hand, the positive control did not differ significantly from the $50 \%$ and $100 \%$ sambong treatment while the negative control $(\mathrm{p}=0.004)$ and $200 \%(\mathrm{p}=0.003)$ sambong treatment showed a significant difference.

Table 3 shows the amount of potassium permanganate $\left(\mathrm{KMnO}_{4}\right)$ titrant used during titration of the left kidney homogenates. From these volumes, the amount of calcium oxalate crystals in the kidneys were obtained and the percent mass of calcium oxalate were calculated. Data showed the lowest mass percentage in the $50 \%$ sambong treatment group.

\section{DISCUSSION}

After induction of urolithiasis in Wistar rats, treatment with $50 \%$ and $100 \%$ sambong fed orally for 21 days showed a significantly lower number and percent mass of calcium oxalate kidney stones compared to the negative control but was comparable to potassium citrate. The mechanism of action of sambong has not been fully elucidated. The anti- urolithiasis activity is probably due to several mechanisms which may include a diuretic effect and a direct chemolytic effect. Sambong has been elucidated to cause diuresis of 600 $\mathrm{mL}$ of urine from baseline after three days of treatment of $32-50 \mathrm{mg} / \mathrm{kg}$ of sambong. ${ }^{10}$ There was a potassium-sparing effect in these patients with minimal decrease in serum potassium levels. Other mechanisms may include direct chemolysis of stones as seen in the experiments of Rico wherein he used a flow by apparatus to compare the effects of urine from those not taking sambong, compared to urine in patients who were dosed with sambong on the dissolution of calcium oxalate stones. ${ }^{11}$ It showed that patients who took sambong at $40 \mathrm{mg} / \mathrm{kg}$ had the highest dissolution rate compared to no intake of sambong.

Another study looked at the effect of B. balsamifera extract on the morphology of calcium oxalate crystals by light microscopy, image analysis, powder $\mathrm{X}$-ray diffraction, and scanning electron microscopy, ${ }^{12}$ exposure to the $B$. balsamifera extract produced a decrease in crystal size by $5.22-82.63 \%$ which differed with the degree of saturation. There was also a shift on the phase of crystals from calcium oxalate monohydrate to calcium oxalate dehydrate, which also increased with the concentration. Scanning electron microscopic images showed discernible crystal unit boundaries with higher concentrations. This further validated the chemolytic effects of $B$. balsamifera.

In this study, the most effective dosages of treatment in inhibiting kidney oxalate stone formation were the 50\% and $100 \%$ sambong treatment. The mean number of oxalate crystals in the said groups was found to be significantly different from the negative control group which was expected to have the greatest number of oxalate crystals. Although the $100 \%$ sambong treatment group had the least number of oxalate crystals, its effect did not differ significantly from the 50\% sambong treatment and the positive control. Thus, it can be said that the anti-urolithiatic effects of the $100 \%$ and $50 \%$ sambong treatment are comparable with the effects of the positive control (i.e. potassium citrate) which is documented in the literature to have anti-urolithiatic effects on individuals with kidney crystals. ${ }^{12}$

The 200\% sambong treatment had the least antiurolithiatic effects. The mean number of oxalate crystals formed in this treatment was not found to be significantly different from the negative control group. It was observed that most rats died in the $200 \%$ treatment group reducing the sample size from $\mathrm{n}=6$ to $\mathrm{n}=3$ and thus can be hypothesized as a toxic dose to the rats. The induction of oxalate crystals using ethylene glycol could also have caused acute renal failure due to its toxicity. During the process of forced feeding, rats in the $200 \%$ sambong treatment group showed an aversion to drinking the treatment. This was also observed in the $50 \%$ and $100 \%$ treatment groups but the rats in the $200 \%$ concentration treatment group were the most difficult to feed. It was surmised that the rats had more difficulty consuming the sambong treatment with higher 
concentrations probably due to the taste of the concentrated solution. This may also be a contributing factor to the trend of decreasing effect with increasing concentration as rats that averted consumption of the concoction are less exposed to the treatment hence less effect was observed.

The results confirm that potassium citrate and the DOH-prescribed 100\% sambong treatment do have antiurolithiatic property. It also shows that giving a dosage of $50 \%$ sambong will have a similar effect as the $100 \%$ concentration and potassium citrate assuming equal volumes. Furthermore, the concentration of the sambong treatment is not directly proportional to its anti-urolithiatic effect with the $100 \%$ sambong treatment group showing the best anti-urolithiatic property.

There is an inverse relationship between the percent mass of calcium oxalate and the effectiveness of the treatment. The 50\% sambong treatment yielded the least percentage mass of calcium oxalate thus making it the concentration with the most anti-urolithiatic effect. Moreover, it is lower than the negative control, which generated the highest percent mass of calcium oxalate.

Limitations in the analysis of the titration are due to the very small dry weight of the samples. Ideally, after oven drying, the amount of dried kidney samples should weigh at least $100 \mathrm{mg}$ per kidney in order to ensure detection of the calcium oxalate crystals through titration. However, the drying process resulted in kidney mass of less than the recommended values. To address this, samples were pooled within the same treatment group, assuming that the level of treatment effects for each rat of the same treatment group were similar. Other limitations include being a post-test only research. Taking histological samples prior to treatment is not possible since this would entail sacrifice of the animals.

Comparing the treatments to the negative control, it is evident that sambong indeed has an anti-urolithiatic capability. The positive control which contains potassium citrate, the $50 \%$ sambong, and the $100 \%$ sambong treatment all have anti-urolithiasis effect based on titration data. This is consistent with the obtained results from the histopathologic analysis of kidneys from the same specimen.

This study warrants more data gathering on sambong extract. Future researches may look for the possible mechanisms of action.

\section{CONCLUSION}

Based on data interpretation and analysis, it can be concluded that Blumea balsamifera (sambong) reduces calcium oxalate stone formation in the kidneys showing its thiazidelike property. The $50 \%$ and $100 \%$ sambong treatment were the most effective concentrations in terms of reduction of the number of crystal formation seen via microscopy while the $50 \%$ sambong treatment yielded the least percent mass of calcium oxalate through titrimetric analysis.

\section{Statement of Authorship}

All authors participated in data collection and analysis, and approved the final version submitted.

\section{Author Disclosure}

All authors declared no conflict of interest.

\section{Funding Source}

This paper was funded by the College of Medicine, University of the Philippines Manila.

\section{REFERENCES}

1. PubMed Health, Kidney stones [Internet]. 2012 [cited 2012 Oct]. Available from http://www.ncbi.nlm.nih.gov/pubmedhealth/ PMH0001493/.

2. WHO, Philippines. [Internet]. 2011 [cited 2012 Oct]. Available from http://www.wpro.who.int/countries/phl/26PHLpro2011_finaldraft. pdf

3. University of Maryland Medical Center, Herbal medicine [Internet]. 2012 [cited 2012 Oct]. Available from http://www.umm.edu/ health/medical/altmed/treatment/herbal-medicine

4. Cuevas et al. ed. DOH handouts on herbal medicine. "Public Health Nursing in the Philippines". Manila: Publications Committee, National League of Philippine Government Nurses, Inc; 2007.

5. de Water R, Boeve ER, van Miert PP, Deng G, Cao LC, Stijnen T, et al. Experimental nephrolithiasis in rats: the effects of ethylene glycol and vitamin D3 on the induction of renal calcium oxalate crystals. Scanning Microsc. 1996; 10(2):591-601.

6. Luna LG. Manual of Histologic Staining Methods of the Armed Forces Institute of Pathology, 3rd ed. New York: McGraw-Hill; 1968.

7. Simon M, Chalfoun J, Brady M, Bajcsy P. Do we trust image measurements? Variability, accuracy and traceability of image features. 2016 IEEE International Conference on Big Data (Big Data). 2016; 1474-82.

8. Abdelkarim AS, Ali M, Naquivi KJ. Antinephrolithiasic activity of Sudanese Trigonella foenum-graecum L. seeds. Asian Pac J Trop Biomed. 2012; 1-4.

9. Ramachandran S, Vijayakumar TM, Saisandeep V, Ramsai K, Dhanaraju MD. Antilithiatic activity of poly-herbal extracts on ethylene glycol-induced lithiasis in rats. Europ J Biol Sci. 2011; 3(2):36-9.

10. De Leon D, Cortes-Maramba NP and the National Integrated Program on Medicinal Plants. Phase I Study: Sambong tablet as a diuretic. In: Technology Transfer Document, Philippine Council for Health Research and Development. 1998.

11. Rico F. Sambong (Blumea balsamifera): Its effect on calcium stone. Philipp J Urol. 1992; 2(1):9-13.

12. Montealegre CM, De Leon RL. Effect of Blumea balsamifera extract on the phase and morphology of calcium oxalate crystals. Asian J Urol. 2017; 4(4):201-7. 